\title{
Live Demonstration: A Wearable Torso Shape Detection Belt for Lung Respiration Monitoring
}

\author{
$\mathrm{Yu} \mathrm{Wu}{ }^{1}$, Dai Jiang ${ }^{1}$, Andy Bardill ${ }^{2}$, Serena De Gelidi ${ }^{2}$, Richard Bayford ${ }^{2}$ and Andreas Demosthenous ${ }^{1}$ \\ ${ }^{1}$ Department of Electronic and Electrical Engineering, University College London, Torrington Place, London WC1E 7JE, UK \\ ${ }^{2}$ Department of Natural Sciences, Middlesex University, The Burroughs, London, NW4 4BT, UK \\ e-mail: yu.wu.09@ucl.ac.uk; a.demosthenous@ucl.ac.uk
}

\begin{abstract}
A 32 channel wearable torso shape detection belt will be demonstrated. The belt is designed to measure the torso shape of a neonate and provide real-time boundary information to assist the electrical impedance tomography (EIT) system to produce high quality lung respiration images. The system is fully integrated on a flexible printed circuit board which is encapsulated in a silicon wearable cover. During the live demonstration, while EIT images are reconstructed, the boundary shape can be changed to improve the image.
\end{abstract}

\section{INTRODUCTION}

Electrical impedance tomography (EIT) is a non-invasive, and radiation-free technique that provide real-time lung respiration images for monitoring aeration in neonate applications. EIT images an object by injecting currents through electrodes on the object's surface and measuring the potential developed on the surface. Algorithms use the recorded voltages to reconstruct an image that represents the distribution of the inner conductivity of the object within the defined boundary outlines.

Ideally, the boundary outlines should be identical to the outline shape of the object (torso) that is being measured, but current EIT imaging often uses a fixed universal torso boundary shape. For lung respiration monitoring, particularly in neonatal applications, because of individual differences and torso deformations when babies are laying in different positions, many studies have suggested the use of a universal boundary causes significant errors in the end results [1], [2]. Acquiring a patient specific boundary shape of the torso could greatly reduce the distorting artifacts in reconstructed EIT images. Some methods have already been proposed [2], [3], [4], but a compact wearable device that can provide an accurate patient specific boundary in real-time does not exist.

In this demonstration, we present a wearable torso shape detection belt that identifies the shape using 32 MEMS sensors [2]. The sensors are mounted on a flexible printed circuit board as shown in Fig. 1(a). Through a signal processing hub, the captured data are sent to a remote computer for boundary shape reconstruction, while EIT also takes place to capture lung respiration data for imaging. The EIT and shape data are combined to reconstruct an EIT image with patient specific boundary information.

\section{DEMONSTRATION AND VISITOR EXPERIENCE}

The demonstration includes the boundary shape belt, an EIT system [3] and a remote laptop computer. The EIT system takes

This work is funded by the European Commission under grant agreement no. 668259 - CRADL (www.cradlproject.org).

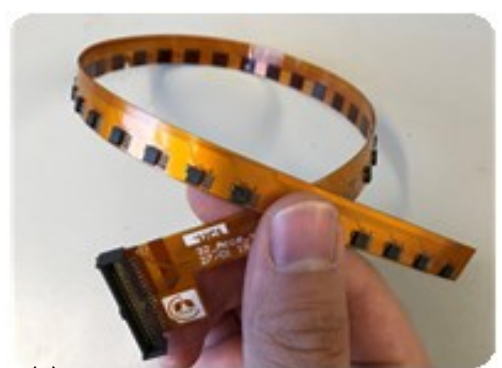

(a)

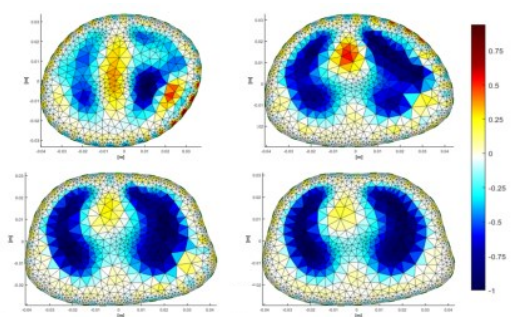

(b)

Fig. 1. (a) Wearable torso shape detection belt. (b) Reconstructed EIT images using the same set of data but with different boundaries shapes to illustrate that an accurate torso boundary shape can significantly improve the quality of lung EIT images.

measurements from an EIT phantom whose outline shape is unknown to the visitor. The visitor will try to wrap the belt into various shapes while the computer reconstructs images using the measured data from the EIT system with the boundary shape information gathered by the belt. As shown in Fig. 1(b), only when the boundary shape gets close to the unknown outline shape of a phantom, its content is revealed to the visitor in the EIT image.

\section{REFERENCES}

[1] J. M. Khor, A. Tizzard, A. Demosthenous, and R. Bayford, "Wearable sensors for patient-specific boundary shape estimation to improve the forward model for electrical impedance tomography (EIT) of neonatal lung function," Physiol. Meas., vol. 35, no. 6, pp. 1149-1161, May. 2014

[2] S. de Gelidi et al., "Torso shape detection to improve lung monitoring," Physiol. Meas., vol. 39, no. 7, pp. 074001-1-074001-11, Jul. 2018

[3] Y. Wu, P. Langlois, R. Bayford, and A. Demosthenous, "Design of a CMOS active electrode IC for wearable electrical impedance tomography systems," in Proceedings - IEEE International Symposium on Circuits and Systems, 2016, vol. 2016-July, pp. 846-849.

[4] Y. Wu, D. Jiang, A. Bardill, S. de Gelidi, R. Bayford and A. Demosthenous, "A high frame rate wearable EIT system using active electrode ASICs for lung respiration and heart rate monitoring," IEEE Trans. Circuits Syst. I: Regular Papers, vol. 65, no. 11, pp. 3810-3820, Nov. 2018 